\title{
Thomas Blanke Die Hartz-Reformen - Kurswechsel im Arbeits- und Sozialrecht
}

\section{Die Vorschläge der»Hartz-Kommission«}

Mitte 2002, rechtzeitig vor Beginn des Bundestagswahlkampfes, wurden der bundesdeutschen Öffentlichkeit die Ergebnisse der »Kommission für moderne Dienstleistungen am Arbeitsmarkt « vorgelegt. ${ }^{.}$Die I 5 -köpfige Kommission war im Februar 2002 ins Leben gerufen worden, nachdem die Bundesanstalt für Arbeit (BA) aufgrund falscher Zahlen und Berichte über die Erfolge ihrer Vermittlungstätigkeit ins Kreuzfeuer der Kritik geraten war. Auftrag der Kommission war es u.a., Vorschläge für grundlegende Reorganisation der BA und eine effektivere Arbeitsvermittlung zu erarbeiten. Sie hat diesen Auftrag, nach den Worten ihres Vorsitzenden, dem Personalchef im Vorstand der VW AG, Peter Hartz, dahingehend genutzt, »dass sie den Abbau von zwei Millionen Arbeitslosen in drei Jahren zum Ziel eines Gesamtkonzepts gemacht hat. $\ll^{2}$ Kernpunkte der Kommissionsvorschläge sind neben der Umorganisation der BA eine Fülle ineinandergreifender Module zur effizienteren Arbeitsvermittlung (JobCenter, Vereinfachung des Leistungsrechts, Meldepflicht bei Kündigungen und Befristungen, Beratungsteams, Einführung von PersonalServiceAgenturen (PSA) in jedem Arbeitsamtsbezirk etc.) und zur Schaffung neuer Arbeitsplätze (Förderung der Beschäftigung in privaten Haushalten, Schaffung von sog. »Ich-AG's « und »Familien-AG's « durch Gewährung von Existenzgründungszuschüssen an Arbeitslose, Job-Floater, Beschäftigungsbilanzen und Prämierung von Beschäftigungssteigerungen). Motto der Kommissionsarbeit war: »Arbeit soll sich lohnen, nicht Arbeitslosigkeit « ${ }^{3}$

Nach ihrer äußerst knappen Wiederwahl im September räumte die Bundesregierung der punktgenauen Umsetzung der Hartz-Vorschläge, von der sie eine Halbierung der Arbeitslosenzahlen erwartete, höchste zeitliche und sachliche Priorität ein. Das Arbeits- und Wirtschaftsministerium wurden zusammen gelegt und ihre Leitung dem Ministerpräsidenten von Nordrhein-Westfalen, Wolfgang Clement, anvertraut. Unter dessen Federführung wurde das Gesetzespaket mit seinen umfassenden Neuregelungen erarbeitet und geradezu in »Rekordzeit« verabschiedet: Erstmals am 5. II. 2002 wurden die in ein nicht zustimmungspflichtiges Erstes und ein zustimmungspflichtiges Zweites Gesetz für moderne Dienstleistungen am Arbeitsmarkt

I Der 353 Seiten umfassende Bericht wurde am I6. 8. 2002 vor 500 geladenen Gästen im Französischen Dom am Berliner Gendarmenmarkt von Bundeskanzler Gerhard Schröder und Volkswagen-Personalvorstand Dr. Peter Hartz der Öffentlichkeit präsentiert. Er steht unter dem Motto: »Eigenaktivitäten auslösen Sicherheit einlösen «; vgl. P. Hartz u. a., Moderne Dienstleistungen am Arbeitsmarkt. Bericht der Kommission (Broschüre A 306 des BMA, Berlin 2002) sowie die zusammenfassende Darstellung, in: Soziale Sicherheit H. 8-9 2002, S. 254 ff.; dort auch eine erste Stellungnahme des DGB mit dem Titel »Ein viel versprechendes Konzept für die Zukunft«, ebd. S. 26I ff.

2 Vorwort von P. Hartz zu dem Bericht, in: Soziale Sicherheit (Fn. I), S. 254.

3 Vorwort von P. Hartz zu dem Bericht, in: Soziale Sicherheit (Fn. I), S. 254. 
aufgespaltenen Gesetzesentwürfe vorgelegt. Der Bundestag stimmte ihnen auf der Grundlage der Beschlussempfehlungen des Ausschusses für Wirtschaft und Arbeit am I 5. I 2. 2002 in dritter Lesung gegen die Stimmen der Opposition zu. Der Vermittlungsausschuss von Bundestag und Bundesrat erzielte am I7. I 2. 2002 Einigung über eine Reihe von Änderungsvorschlägen zu beiden Gesetzen. ${ }^{4}$ Diese wurden am 20. I 2. 2002 von Bundestag und Bundesrat beschlossen und am 23. I2. 2002 im Bundesgesetzblatt verkündet.

Unter demokratietheoretischen wie -praktischen Gesichtspunkten sind es insbesondere zwei Elemente, die an dieser Vorgeschichte der umfassendsten Arbeitsmarktreform der vergangenen zwanzig Jahre nachdenklich stimmen: Einmal ihre Vorbereitung durch eine (parlaments-)unabhängige Sachverständigenkommission, deren Vorschläge sogleich mit der Ankündigung der Öffentlichkeit präsentiert werden, an ihnen werde kein Jota mehr geändert.'

Und zum andern die Gesetzgebungshektik, die es (wohl außer den Mitgliedern des zuständigen Parlamentsausschusses und einem engsten Kreis von Politikberatern und Verbandsfunktionären) allenfalls noch versierten Internetvirtuosen ermöglichte, auch nur mit dem äußeren Gang der parlamentarischen Beratungen Schritt zu halten. Für die Möglichkeit, die Gründe und Gegengründe für die vorgeschlagenen Neuregelungen zur Kenntnis zu nehmen und halbwegs in Ruhe gegeneinander abzuwägen, blieb keine Zeit.

Beide Elemente, die autoritär verordnete Kommissionsdemokratie und die überfallartige Gesetzgebungsdynamik, verkürzen das Recht der politischen Öffentlichkeit, zu der nach Lage der Dinge auch die Verbände gehören, auf Mitwirkung an der politischen Willensbildung und entwerten den parlamentarischen Gesetzgeber, indem sie ihn vor vollendete Tatsachen stellen. Dieses Vorgehen erklärt sich aus dem entschlossenen Handlungswillen einer Regierung, die keinen anderen Ausweg mehr sieht, um eine wahrgenommene Notlage zu wenden. Hinzu kommt, dass der für erforderlich gehaltene Kurswechsel offenbar als derart grundlegend eingeschätzt wird, dass er zerredet und zerrieben werde, wenn das Schicksal des Reformprojekts den mächtigen Interessengruppen der Verbändedemokratie und ihren parlamentarischen Repräsentanten überantwortet würde.

Die schlechten Zeugnisse, die die Medien der Startphase der neuen Bundesregierung ausstellten, wurden immer wieder auch damit begründet, dass es dem Kanzler nicht gelungen sei, die Ergebnisse der »Hartz-Kommission« wirklich I: I umzusetzen. Dadurch seien die mutigen Reformvorhaben, insbesondere unter dem Einfluss der Gewerkschaften, verwässert und um ihre mutmaßliche Wirkung gebracht worden. Das breite Publikum, soviel scheint diesen Einschätzungen zu entnehmen zu sein, will noch weniger Öffentlichkeitsbeteiligung. Damit kehrt sich im Ergebnis die von der Bundesregierung forcierte Entwertung der öffentlichen Auseinandersetzung und der kontroversen parlamentarischen Debatte gegen sie selbst. Das Echo auf den Ruf nach dem starken Arm erschallt immer von der Gegenseite.

4 BT-Drs. I 5/202 v. I7. I0. 2002; vgl. hierzu auch die Pressemitteilung pr253_02.html auf der homepage des Deutschen Bundesrats.

5 Dies war angesichts der Konkretisierungsbedürftigkeit der Kommissionsvorschläge »naiv, ja sinnlos «, so R. Hickel, Hartz-Konzept: Arbeitslose effektiver in billige Jobs - Deregulierungsschub auf den Arbeitsmärkten, in: Aus Politik und Zeitgeschichte. Beilage zur Wochenzeitung Das Parlament, 6-7/2003 v. 3. 2. 2003 , S. 7 ff., 9. Seine Forderung nach einem »breiten gesellschaftlichen Dialog darüber, ob die Gesellschaft sich zu solcherart massiven Veränderungen durchzuringen vermag, « ist von der Realität längst überholt. 
Mit dem Ersten und Zweiten Gesetz für moderne Dienstleistungen am Arbeitsmarkt vom 23. I 2. $2002^{6}$ sind die Vorschläge der sog. Hartz-Kommission ${ }^{7}$ in ein umfangreiches gesetzliches Reformpaket gegossen und verkündet worden. Die neuen Regelungen treten $\mathrm{zu}$ unterschiedlichen Zeitpunkten in Kraft: überwiegend zum I. I. $2003,{ }^{8}$ teils zum April, I. 5. 2003 oder zu einem noch später liegenden Zeitpunkt. ${ }^{9}$ Die Gesetze zur organisatorischen Neuordnung der Bundesanstalt für Arbeit einschließlich der Abschaffung der Landesarbeitsämter und zur Verzahnung der Arbeitslosen- mit der Sozialhilfe sind noch in Vorbereitung und werden für 2004 erwartet. ${ }^{10}$

Arbeitsmarktpolitische Instrumentarien und ihre gesetzliche Normierung besitzen eine beängstigend kurze Halbwertzeit. Das SGB III von I 997 ist allein bis Mitte 2002 nicht weniger als $38 \mathrm{mal}$ novelliert worden, zuletzt durch die umfassenden Neuregelungen in Zusammenhang mit dem I. I. 2002 in Kraft getretenen »Job-AQTIVGesetz «. ${ }^{11}$ Kaum sind die veränderten Bestimmungen verabschiedet und die institutionellen Strukturen zur Durchführung der Maßnahmen aufgebaut, da kündigt sich bereits der nächste Wechsel der arbeitsmarktpolitischen Strategien an. Folge dieser Kurzatmigkeit ist, dass eine erhebliche Forschungslücke in Bezug auf die praktische Wirksamkeit der jeweils favorisierten arbeitsmarktpolitischen Instrumentarien klafft. Seriöse Untersuchungen, welche Effekte welche Programme und die auf ihrer Basis durchgeführten Fördermaßnahmen besessen haben, sind schon auf Grund ihrer zumeist relativ knappen Implementationsphase vergleichsweise selten. Dies ist umso problematischer, als jedes neue arbeitsmarktpolitische Konzept mit dem Versprechen antritt, besser als die zuvor erprobten zur Bewältigung der jeweils anvisierten Beschäftigungsproblematik geeignet zu sein. Auf diese Weise ist in die Abfolge der Instrumentarien ein Automatismus des Veraltens eingebaut, der es als unwahrscheinlich erscheinen lässt, dass auf die früheren, als »verbraucht« angesehenen Konzepte noch zurückgegriffen werden kann. Ein derartiger Politikmodus der rapiden Entwertung vergangener Konzepte ist insbesondere dann hochgradig problematisch, wenn dieser Beurteilung keine gesicherten empirischen Kenntnisse über die Effektivität der abgelösten - und keine entsprechend fundierten Annahmen hinsichtlich der voraussichtlichen Wirksamkeit der jeweils neueren - Maßnahmen zugrunde liegen. Wissenschaftlich seriöse Implementationsforschung ist das Hauptdesiderat jeder gezielt regulativen Maßnahmegesetzgebung.

Dieser Generalvorbehalt gilt auch für die aktuellen Gesetze für moderne Dienstleistungen am Arbeitsmarkt. Aber er trifft sie in anderer Weise als frühere Gesetzes-

6 BGBl. I 2002 S. 4607 und S. 462 I.

7 Die Kommission »Moderne Dienstleistungen am Arbeitsmarkt« war im Frühjahr 2002 von der Bundesregierung eingesetzt worden, um insbesondere Vorschläge zur Verbesserung der Arbeitsmarktchancen von Arbeitssuchenden und eine raschere Vermittelbarkeit von Arbeitslosen zu erarbeiten. Kurz zuvor, zum I. I. 2002, war das sog. "Job AQTIV «-Gesetz (v. IO. I 2. 200I, BGBl. I S. 3443) in Kraft getreten, welches ebenfalls auf eine grundlegende Reform der Arbeitsmarktpolitik zielte. Zwischen beiden Maßnahmen war die Bundesanstalt für Arbeit (BA) in die Schlagzeilen der Kritik geraten, weil sich die von ihr gemeldeten Vermittlungszahlen als überhöht erwiesen hatten, und ihre organisatorische Umgestaltung eingeleitet worden.

8 Art. I 4 Abs. I Erstes und Art. I7 Abs. I Zweites Gesetz für moderne Dienstleistungen am Arbeitsmarkt.

9 Dies gilt insbesondere (mit Ausnahme des neu gefassten $\$ I I AÜG) für die Änderungen des Arbeitnehmerüberlassungsgesetzes. Diese gelten für Leiharbeitsverhältnisse, die vor dem I. I. 2004 begründet worden sind, sofern für diese kein spezieller Tarifvertrag eingreift, der zumindest die in $\int \mathbb{S} 3 \mathrm{Abs}$. I Nr. 3 und 9 Nr. 2 AÜG bestimmten Mindestarbeitsbedingungen regelt, nach Art. 6 Nr. $1 \circ$ Erstes Gesetz (\$ I 9 Abs. I und 2 AÜG n. F.) bis zum 3I. I 2. 2003 weiter.

Io Vgl. den Bericht in FAZ v. 27. I. 2003, S. 9.

I I BGBl. I S. 3443 . 
novellen. Denn sie markieren nicht nur ihrem Umfang und ihrer Bedeutung nach die wohl seit Jahren einschneidendste Veränderung auf dem Gebiet des Arbeits- und Sozialrechts, sondern sie signalisieren zugleich einen grundlegenden Wechsel der arbeits- und sozialrechtlichen Zielvorstellungen, der die ausgetretenen Pfade tradierter arbeitsmarktpolitischer Instrumentarien verlässt. In ihnen kommt ein verändertes arbeits- und sozialrechtliches Konzept zum Ausdruck, welches erstmals in dieser Deutlichkeit eine neue Handschrift rot-grüner Reformvorstellungen offenbart. Perspektivisch, so die hier vertretene These, läuft dies Konzept auf eine Abkehr von den tradierten, für das vergangene 20. Jahrhundert charakteristischen Zielvorstellungen der Arbeits- und Sozialpolitik hinaus. Dies hat eine Veränderung ihrer grundbegrifflichen Definitionen und der in diese eingeschlossenen Orientierungen - wie etwa dem Arbeitnehmer- und Beschäftigtenbegriff, der Bewertung sog. atypischer Beschäftigung, dem Verständnis der Sozialversicherung und der Tarifautonomie - zur Folge und markiert damit eine rechtspolitische Kehrtwendung, die ihren Niederschlag mehr als nur ansatzweise bereits in den jetzt beschlossenen Gesetzen gefunden hat.

Der national geprägte Sozialstaat des 20. Jahrhunderts geht zur Neige. Er weicht einem Konzept moderner Wohlfahrtsstaatlichkeit, dessen zentrale Elemente - Erweiterung des Arbeitsbegriffs, Abkehr von der arbeits- und sozialrechtlichen Zentralität des Normalarbeitsverhältnisses, Aufhebung der scharfen rechtlichen Schwelle zwischen Arbeitnehmern und Selbständigen, Ausdehnung der Sozialversicherungspflicht auf »abhängige« und kleine Selbständige, Aufbau privater Zusatzversicherungen bei Einschränkung der paritätischen Beitragsfinanzierung und stärkerer Steuerfinanzierung in Bezug auf die Sozialversicherung, Einführung einer allgemeinen Grundsicherung etc. - schon seit längerem in Fachkreisen diskutiert wurden ${ }^{12}$ und Eingang insbesondere in die sozialpolitische Programmatik der GRÜNEN und von Teilen der CDU (Biedenkopf, Miegel) gefunden hatten.

Dass wesentliche Anfangsschritte zur Umsetzung dieser Programmatik ausgerechnet in eine Zeit ökonomischer Depression mit nachlassendem Wirtschaftswachstum, verstetigter und anhaltend steigender Massenarbeitslosigkeit sowie knapper fiskalpolitischer Handlungsspielräume der öffentlichen Kassen fallen, mag sich daraus erklären, dass ohne eine solche Dramatik der nunmehr erfolgte Kurswechsel nicht durchsetzbar gewesen wäre. Er erhält zugleich Rückenwind durch die vermehrten Kompetenzen und Aktivitäten auf europäischer Ebene, die ohnehin auf eine Abkehr von nationalstaatlichen Traditionen drängen. Dass er sich als sozialpolitische Notstandsmaßnahme legitimiert und rechtfertigen muss, ist aber mehr als nur ein Schönheitsfehler dieses ersten größeren Entwicklungsschrittes hin zu einem gewandelten Konzept von Sozialpolitik. Dadurch haftet ihm von Beginn an zugleich der bittere Beigeschmack von Leistungskürzungen auf Kosten derjenigen an, die sich am wenigsten wehren können. ${ }^{13}$

Nachstehend stellen wir, aus der Feder unterschiedlicher Autorinnen und Autoren, die wesentlichen Schwerpunkte der Gesetzesnovelle dar und unterziehen die Veränderungen einer ersten rechtlichen und rechtspolitischen Bewertung und Einschätzung: Die Neuregelungen zur Leiharbeit und zur Vermittlungsaufgabe der mit Hilfe der Arbeitsämter zu errichtenden »Personal-Service-Agenturen« behandelt Wolfgang Däubler. Karl-Jürgen Bieback analysiert die sozialrechtlichen Auswirkungen der auf diesem Gebiet besonders zahlreichen Gesetzesänderungen. Dagmar Schiek untersucht, ob und inwiefern die Neuregelungen insbesondere zur Teilzeitarbeit und

I 2 Grundlegend: Opielka/Ostner (Hrsg.), Umbau des Sozialstaats, I987; zu den alternativen Konzepten des bundesdeutschen Wohlfahrtsstaats vgl. Bleses/Seeleib-Kaiser, Zum Wandel wohlfahrtsstaatlicher Sicherung in der Bundesrepublik Deutschland: Zwischen Lohnarbeit und Familie, ZfS I 999, S. I I 4 ff.

I 3 Däubler, AiB 2002, 729 ff., 735 . 
Befristung sowie zur Leiharbeit europarechtlichen Vorgaben folgen bzw. ihnen genügen. Und Thomas Blanke thematisiert die Wandlung des Beschäftigten- und Arbeitnehmerbegriffs sowie die geänderten Regelungen zur geringfügigen Beschäftigung. 